Perspectiva Geográfica ISSN 0123-3769 Vol. 15/2010; pp. 201-224

\title{
Alternativas de planificación de las actividades productivas primarias en el marco del desarrollo territorial sostenible en la Patagonia Sur Argentina
}

Alternatives for planning of primary production activities within the framework of the sustainable spatial development in Southern Patagonia Argentina

\author{
Carla Narbaiza* \\ Alejandro Schweitzer ${ }^{* *}$
}

\section{Resumen}

Las actividades productivas primarias que se desarrollan en el territorio de la Patagonia Sur argentina (ganadería, minería, actividad forestal y agricultura) no han podido sostenerse sin causar desertificación, contaminación y sobreexplotación de los bosques nativos, que ponen en riesgo el potencial desarrollo de la región. El objetivo deltrabajoes presentar alternativas de planificación territorial de las actividades productivas primarias en la Patagonia Sur argentina, que sirvan de apoyo al proceso integral de toma de decisiones en el marco del desarrollo territorial sostenible. Utilizando una metodología aplicada mixta (documental y de campo) y descriptiva longitudinal (retrospectiva y prospectiva), se propuso un escenario tendencial, que se presenta como un futuro poco alentador, y dos alternos, uno que considera una situación futura muy negativa, y otro

* Magíster en Geografía. Investigadora externa. carlanarbaiza@gmail.com

* Doctor en Geografía. Docente e investigador Universidad Nacional de la Patagonia Austral. alejandro.schweitzer@gmail.com 
que considera el camino más adecuado para lograr un Desarrollo Territorial Sostenible, que se podrá alcanzar, dadas las potencialidades de la región, si se aúnan intereses en forma solidaria.

Palabras clave: Desarrollo Territorial Sostenible, Región Patagonia Sur, Planificación de actividades productivas primarias, Prospectiva.

\begin{abstract}
The primary productive activities in the territory of the argentinian southern Patagonia (livestock, mining, forestry and Agriculture) not have been able to be sustainable without causing desertification, pollution and overexploitation of native forests, threatening the development potential of the region. This work aims to present alternatives in territorial planning of the primary productive activities in the argentineian southern Patagonia so they can serve as a support to the integrated process of decision making in the framework of sustainable territorial development. Using an applied mixed methodology (documentary and field) and descriptive longitudinal (retrospective and prospective), a trend scenario was proposed, which is presented as a very little encouraging future, and two alternate, one that considers a very negative future situation, and one that considers the most appropriate way to achieve a sustainable Territorial Development, which can be reached given the potential of the region if interests are combined in a solidary way.
\end{abstract}

Key words: Sustainable Territorial Development, Southern Patagonia Region, Planning of primary production activities, Foresight. 


\section{Introducción}

El ser humano, al igual que los restantes seres vivos, forma parte de la naturaleza, a la cual está unido por múltiples vínculos de interdependencia, y se integra a los ecosistemas, principalmente, de dos maneras: por las extracciones sobre el ecosistema, como la utilización del aire, el agua y los minerales, por ejemplo, y por las modificaciones a dichos ecosistemas (Tricart y Kilian, 1982), con las diferentes actividades productivas $\mathrm{y}$ contaminaciones de todo tipo.

Las actividades productivas primarias son aquellas que crean utilidad a través del aprovechamiento directo de los recursos de la naturaleza para obtener beneficios y satisfacer necesidades humanas; se incluyen en ellas la agricultura, la ganadería, el aprovechamiento forestal, la pesca, la minería metalífera (oro y plata, por ejemplo) y no metalífera, como el carbón, así como también la extracción de hidrocarburos como el gas y el petróleo.

Una actitud racional consistiría en efectuar sobre los ecosistemas las extracciones máximas que puedan soportar sin degradarse ni destruirse; esto responde a proteger el futuro y perpetuar la especie humana. Sin embargo, efectuar una explotación razonable de los ecosistemas sin degradarlos ni superar su umbral de sensibilidad, no es tarea fácil. En primer lugar, hay que adaptarse a la diversidad de los ecosistemas en los que humanidad inserta sus actividades (Tricart y Kilian, 1982), y luego tener en cuenta que las actividades productivas primarias suelen presentar conflicto entre los actores interesados.

Desde la geografía, la forma de tratar dicha problemática dentro de la teoría de sistemas es mediante el análisis integral y dinámico del paisaje. Las unidades de paisaje resultan como una expresión integrada de componentes físicos, biológicos y culturales que se presentan en el espacio geográfico con una fisonomía particular (Méndez, 2006) y pueden ser utilizadas para la ordenación del territorio y el desarrollo sustentable (Naranjo, 2006; López y Cervantes, 2002).

Reátegui (2008) afirma que el desarrollo es más que la ausencia de subdesarrollo y más que la aspiración de crecimiento como un fin; sostiene que al desarrollo hay que entenderlo como proceso de cambios cualitativos y transformaciones de las estructuras económicas, sociales y políticas, en armonía con los sistemas naturales. Por lo tanto, en el desarrollo territorial sostenible la idea de futuro a largo plazo requiere prever con prudencia, imaginación, creatividad y audacia lo que puede suceder. Es así como el desarrollo y la articulación de la prospectiva territorial permiten a los actores prepararse para el cambio y provocarlo (Troitiño, 2006). El futuro es "fruto de nuestro esfuerzo y de nuestro sacrificio. En definitiva, de nuestra ambición y de nuestra voluntad" (Gabiña, 1999).

Sin embargo, en Latinoamérica la mayoría de los países encuentran dificultades para impulsar proyectos de desarrollo autónomo, muchas veces a 
causa de la debilidad en políticas orientadas al desarrollo sostenible, y a la dependencia de créditos externos (MaxNeef, Elizalde, Hopenhayn, 1996); por lo tanto, se ven limitados en la adaptación de estrategias progresistas, y como consecuencia viven el cortoplacismo para la resolución de los problemas (Massiris, 2006; Gabiña, 1999). A su vez, en estos tiempos en que los ritmos de cambio son más rápidos, los mecanismos de respuesta de la sociedad resultan lentos. Por lo tanto, es necesario adelantarse y prever para introducir medidas correctivas (Gabiña, 1999) que permitan planificar a largo plazo un futuro deseable y sostenible, cuidando los recursos naturales y mejorando la calidad de vida de la población.

Dentro del análisis regional, la teoría de sistemas realiza un aporte teórico interesante, donde la región se entiende en forma integral. García (2006: 49), haciendo uso de la palabra de Nir (1990), define la "región sistémica" como: "una porción de la superficie terrestre donde la población, organizada en el seno de ciertos límites administrativos, políticos o sociales, encara unos retos naturales, sociales, políticos y económicos", destacándose la voluntad holística e integradora de esta visión de región.

De Jong (2009) plantea un análisis regional con una concepción metodológica dirigida a la comprensión del conflicto social y su expresión territorial en el marco de la producción del espacio, más que al abordaje meramente empírico y descriptivo adoptado generalmente. Autores como el citado y Sánchez (1991) plantean que la naturaleza y el espacio son el insumo básico de la reproducción del todo social, siendo este un proceso histórico y dialéctico. En esta dialéctica de las relaciones entre los componentes físicos $\mathrm{y}$ sociales es posible construir un conocimiento prospectivo que aporte a la transformación social.

La noción de territorio, por lo tanto, debe trascender lo espacial, para que así sea considerado un producto social e histórico, resultado de relaciones complejas entre factores naturales, económicos, sociales, culturales y técnicos, formas de producción y una red de instituciones (Troitiño 2006; Sepúlveda, Rodríguez, Echeverri y Portilla, 2003). El territorio es el escenario donde acontecen todas las relaciones sociales, y es imprescindible el papel que juega cuando se trata de impulsar actividades económicas y mejorar el nivel de vida de la población a partir del uso racional de sus propios recursos (económicos, humanos, ambientales, institucionales, culturales) (Troitiño, 2006) y potencialidades, ya que el territorio se construye a partir de la actividad espacial de agentes que operan a diversas escalas, por lo que asume la existencia de un espacio geográfico y un sujeto que ejerce sobre él una relación de poder, una facultad de apropiación (Hernández, 2009), que se hace fundamental armonizar con el tipo de desarrollo que se proponga.

En el territorio de la Patagonia Sur argentina (provincias de Santa Cruz y de 
Tierra del Fuego) las actividades productivas primarias no han podido sostenerse sin causar graves daños ambientales e incompatibilidades marcadas, que están poniendo en riesgo el potencial para el desarrollo de la región. Los conflictos más relevantes se dan por la sobreexplotación de recursos, como la desertificación (Programa de las Naciones Unidas para el desarrollo, 2009; Paruelo y Aguiar, 2003, en: Paruelo, Gollusciol, Jobbágy, Canevari y Aguiar, 2006; del Valle, Elisalde, Gagliardini, Milovich, 2004) y la disminución de las reservas forestales (Collado, 2007), la introducción (Collado y Farina, 2006; Collado, 2007) e invasión de especies exóticas (Cipriotti, Collantes, Rauber, Escartín, Braun, Livraghi, 2008), los impactos sobre la biodiversidad y sus respectivas consecuencias (Schiavini, 2007: 798; Borla y Vereda, 2006), la contaminación por la utilización de cianuro en las actividades mineras, que además exigen un alto consumo de agua, y, como consecuencia de estos daños, conflictos sociales (Schweitzer, 2008).

Sumado a lo anterior, hasta el año 2005 se notaba una falta de continuidad en las políticas de orden territorial y en las políticas regionales puestas en marcha desde el sector público en sus distintas escalas; situación que podría caracterizarse como de "territorios a la deriva", "sin futuro", sin proyecto, como lo expone Schweitzer (2004) en su trabajo "Espacios de planificación y territorios a la deriva en la provincia de Santa Cruz". Un cambio positivo se evidencia a partir de la Política Nacional de Ordenamiento y Desarrollo Territorial, puesta en marcha desde el sector público nacional y acompañada desde las provincias a partir de finales de ese mismo año.

En la región, las actividades productivas primarias han sido esenciales para su desarrollo desde los comienzos del poblamiento, a mediados de siglo XIX. La ganadería ovina extensiva es una de las actividades primarias tradicionales de la región (Quargnolo, 2007), alcanzando en Santa Cruz, en 1952, su máximo valor, 9.105.000 de cabezas de ganado ovino (Williams, 2006), mientras que Tierra del Fuego alcanzaba su máxima producción en 1975, sin llegar al $10 \%$ de la carga animal que Santa Cruz logró en 1952. Con el correr de los años sobrevino una crisis que está llevando prácticamente a la desaparición de la explotación ovina extensiva (Andrade, 2005). En 50 años, Santa Cruz disminuyó en casi el $80 \%$ las existencias máximas alcanzadas; en el año 2000 no llega a los dos millones de cabezas, y se registran numerosas empresas quebradas y establecimientos abandonados (Williams, 2006; Andrade, 2005). Diversos factores han motivado la pérdida de rentabilidad del sector lanero y la continua disminución de stocks, entre los más renombrados están los fenómenos climáticos adversos (grandes nevadas, vientos y sequías), pérdidas por acción de depredadores (zorros y pumas, entre otros), deterioro de los recursos forrajeros (desertificación) y disminución de precios de la lana (Williams, 2009; Andrade, 2010). 
Así como la ganadería ovina fue la actividad que impulsó la ocupación del territorio Austral, la actividad hidrocarburífera tuvo un importante impacto en las poblaciones patagónicas, desde el punto de vista histórico y sociolaboral, a partir del descubrimiento del petróleo en Comodoro Rivadavia, entre inicios y mediados del siglo xx (Sarobe, 1999).

La región cuenta con dos cuencas hidrocarburíferas: la del Golfo San Jorge, que está situada al noreste del territorio de Santa Cruz, y la Cuenca Austral, ubicada al sur del continente (Martínez Llaneza, 2010; Equipo de Gestión Económico y Social, [EGES] 2008). La primera aporta cerca del $50 \%$ de la producción del crudo argentino, extrae diariamente más de $46.000 \mathrm{~m}^{3}$, posición que será mantenida en los próximos 10 años, aproximadamente; mientras que la cuenca Austral aporta diariamente unos $5.800 \mathrm{~m}^{3}$ de crudo, y cuenta con reservas para hacerlo durante 6 años más, aproximadamente (Instituto Argentino del Petróleo y del Gas, 2010). Además, esta última aporta el $20 \%$ del volumen de gas natural producido a nivel nacional, que alcanza unos 140 millones de $\mathrm{m}^{3}$ diarios (Martínez Llaneza, 2010).

Los ingresos que generan las regalías del sector energético, gas y petróleo, resultan ser las fuentes más importantes de provisión de recursos financieros a las provincias patagónicas australes (Santa Cruz. Agencia de Desarrollo de Inversiones, 2004). A su vez, este sector, junto con la minería, es una fuente importante de generación de empleos para la región; en la provincia de Santa Cruz, el sector minería y petróleo es responsable del $14,2 \%$ de los empleos, con alrededor de 8.000 puestos de trabajo; mientras que en Tierra del Fuego el mismo sector genera alrededor de 1.000 puestos de trabajo, sin alcanzar a representar el 4\% provincial (Argentina. Ministerio de Relaciones Exteriores, Comercio Internacional y Culto. Subsecretaría de Desarrollo de Inversiones. Prosperar, 2010).

Tradicionalmente, la minería santacruceña se circunscribía a la extracción de carbón, arcillas y caolines, y a la explotación de salinas de cosecha (sal común) para el mercado doméstico. Desde el año 1999 fue puesta en producción la Mina Cerro Vanguardia, destinada a la explotación de oro y plata, la cual mantiene una producción dirigida totalmente al mercado externo; en el 2004 exportó 115 millones de dólares al Reino Unido y Sudáfrica (EGES, 2008).

Otras actividades primarias desarrolladas en la región Patagonia Sur son la producción bovina, que presenta un atractivo horizonte, dada la posibilidad de exportar a países del circuito no aftósico (Schorr y Segui, 2008), así como la producción agrícola, que se desarrolla principalmente en los valles, bajo riego, y en los valles cordilleranos, con cultivos forrajeros y en menor proporción con cultivos fruti-hortícolas; estos últimos tienen un importante potencial exportador, no incentivado adecuadamente (Schorr y Segui, 2008). 
En cuanto al recurso forestal nativo, vale decir que gran parte de Santa Cruz se encuentra en áreas protegidas o de conservación (Schweitzer, 2009), y que cuenta con la menor representatividad superficial y la menor participación en el Producto Bruto Regional, si se comparan las provincias patagónicas. La situación es diferente en la provincia de Tierra del Fuego, donde 713 mil hectáreas se encuentran ocupadas por bosques, de los cuales, aproximadamente, el $30 \%$ corresponden a bosques productivos.

\section{Planteamiento del problema}

En la Patagonia Sur argentina se observa la falta de planificación integral del territorio y una explotación inadecuada de los recursos naturales por parte de las actividades productivas primarias, que condicionan el desarrollo territorial sostenible. Considerando la situación problemática y la riqueza teórica de la geografía, el objetivo general del presente trabajo es proponer alternativas de planificación territorial de las actividades productivas primarias en la región Patagonia Sur argentina, que sirvan de apoyo al proceso integral de toma de decisiones en el marco del desarrollo territorial sostenible. Para lograrlo, primero se realizó una descripción de los procesos territoriales que llevaron a las actuales configuraciones espaciales en la región Patagonia Sur argentina; luego se determinó la oferta de los recursos naturales del área en estudio, así como el impacto que tienen las actividades productivas primarias en el territorio, $y$ el potencial de uso productivo para la región; se identificaron los actores presentes en el territorio, $y$, finalmente, se elaboraron escenarios: uno tendencial y dos alternos: uno de tendencia negativa y otro positiva.

\section{3. Área de estudio}

La región Patagonia Sur (Figura 1) se ubica en el extremo sur del continente americano, y se encuentra compartida por Chile y Argentina. Del lado argentino hace referencia específicamente a las provincias de Santa Cruz y Tierra del Fuego, Antártida e Islas del Atlántico Sur. $\mathrm{Su}$ ubicación geográfica va de los $46^{\circ}$ hasta los $55^{\circ}$ de latitud sur y desde los meridianos $73^{\circ} 29^{\prime}$ hasta los $57^{\circ} 40^{\prime}$ de longitud oeste. En conjunto, ambas provincias tienen una superficie de 265.514 de $\mathrm{km} 2$, con 398.714 habitantes (Instituto Nacional de Estadística y Censos, 2010), sin contar las Islas del Atlántico Sur (Islas Malvinas, Islas Georgias del Sur, Islas Sandwich del Sur e Islas Orcadas del Sur), que en conjunto tienen una superficie de $16.027 \mathrm{~km}^{2}$, y la Antártida argentina, que posee una superficie de $964.847 \mathrm{~km}^{2}$, áreas que no fueron consideradas dentro del área de estudio. 


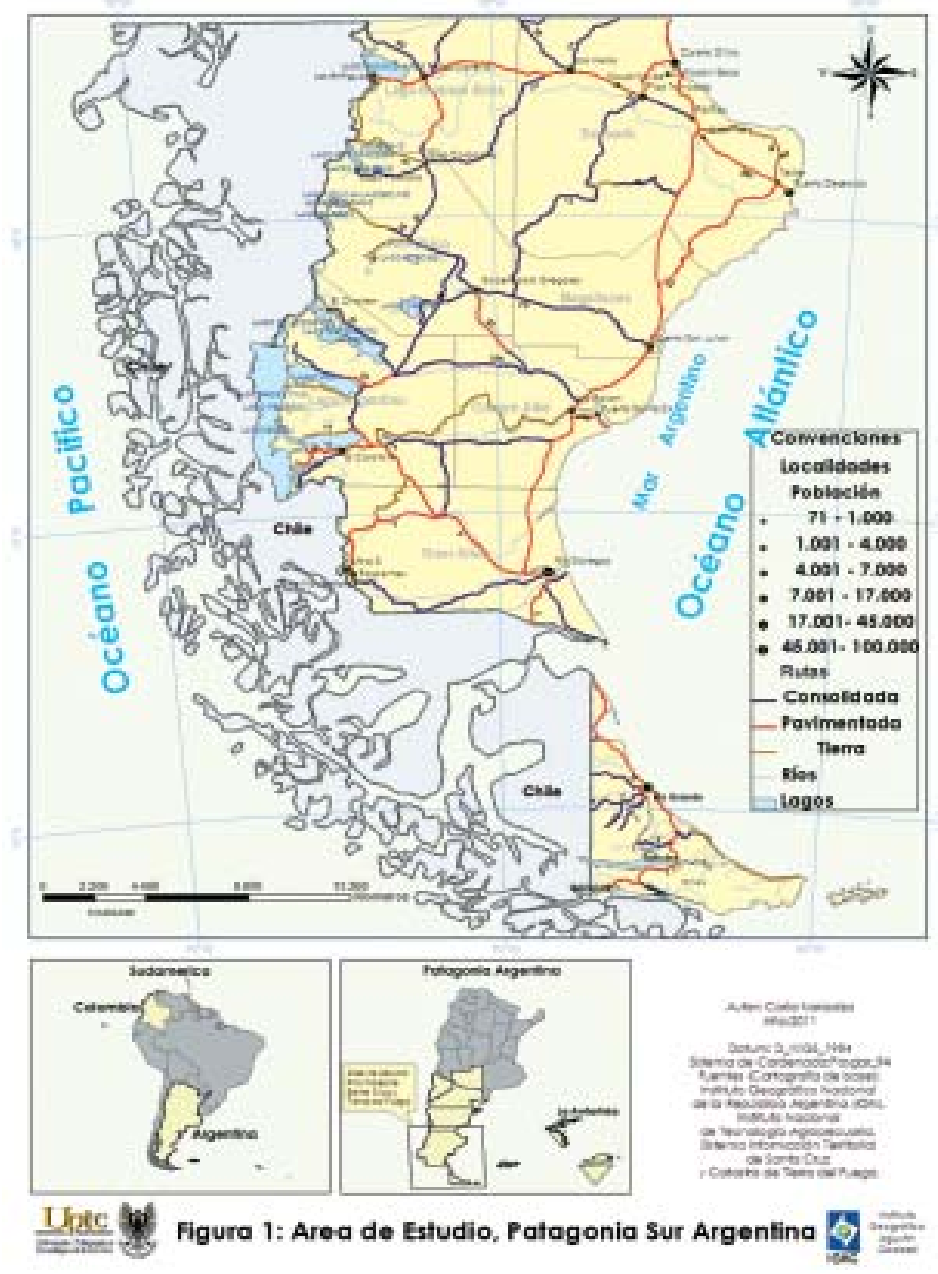

\section{Metodología}

Se utilizó una metodología aplicada mixta (documental y de campo) y descriptiva longitudinal (retrospectiva y prospectiva). Para la búsqueda de las fuentes de información se visitaron 12 instituciones en toda la región y se dialogó en total con más de 60 expertos en las diferentes áreas de interés (directores, subdirectores, secretarios de gobierno, investigadores y extensionistas, y productores). Se envió por correo electrónico una planilla de consulta a 40 de los entrevistados, con el fin de conocer las opiniones de los diferentes actores.

Para la obtención de los escenarios, primero se elaboró la Matriz relacional de análisis estructural - "MotricidadDependencia"-, propuesta por Mojica (2005). Las variables contenidas en la 
Matriz surgen de una lista de "variables relevantes" obtenidas mediante el análisis retrospectivo y actual del área de estudio, en conjunto con la información obtenida de las entrevistas y de las respuestas de los actores principales. En esta Matriz relacional, setenta variables fueron sometidas al «análisis estructural», técnica que está basada en leer la realidad como un sistema, una estructura y un fenómeno complejo, mediante el establecimiento de las relaciones de causalidad entre las diferentes variables, verificando si una variable está afectando a otras.

El análisis estructural tiene en cuenta los conceptos de Motricidad y Dependencia; mientras la primera significa la influencia que una variable ejerce sobre las demás, la segunda representa cuánto se encuentra influenciada una variable por las demás, es decir, qué tan dependiente de las demás variables es. De esta manera, se determinó cuál es la influencia que tiene cada una de las setenta variables sobre las demás, asignando los siguientes valores según correspondiera: $5=$ influencia fuerte; $3=$ influencia moderada; $1=$ influencia débil $; 0=$ influencia nula, y $4=$ influencia potencial. La sumatoria por filas da el valor de Motricidad para la variable "A", y la sumatoria por columna le da el valor de Dependencia a la misma variable "A", formando así con estos valores coordenadas que pueden ser ubicadas en un eje cartesiano como pares de puntos para cada variable.

El gráfico cartesiano (Figura 2) es dividido en cuatro por un valor promedio, que se calcula de la sumatoria de motricidad o dependencia, dividida la cantidad de variables (en este caso setenta), y resulta de determinar el valor que tendría cada variable en el caso de que fuera totalmente independiente. En cada cuadrante hay variables según su valor de motricidad-dependencia, analizadas de la siguiente manera:todas las variables de la Figura 3 influyen mucho sobre las demás, y a su vez son poco influidas por el resto de las variables. Estas variables de producción, económicas y sociales en la zona de poder, están dominando fuertemente el tipo de desarrollo que se da en la región.

La demanda de producción primaria es la principal limitante en un sistema de aprovechamiento de los recursos naturales sin un posterior agregado de valor y de optimización de la cadena productiva a través de tecnologías que sean más amigables con una explotación sostenible, lo que afecta también la estabilidad económica, muy dependiente de las exportaciones y del mercado externo. La ética se presenta como imprescindible para poder encarar cualquier cambio en el sistema productivo, en el se puedan dar las necesarias asociaciones entre productores y se mantengan en el tiempo, sin riesgos de cambiar el rumbo al objetivo principal de desarrollo territorial por eventualidades adversas en el camino.

A su vez, están faltando escuelas de oficio que permitan potenciar las capacidades para el cambio social y productivo de la región. El dominio de las tierras se presenta como 


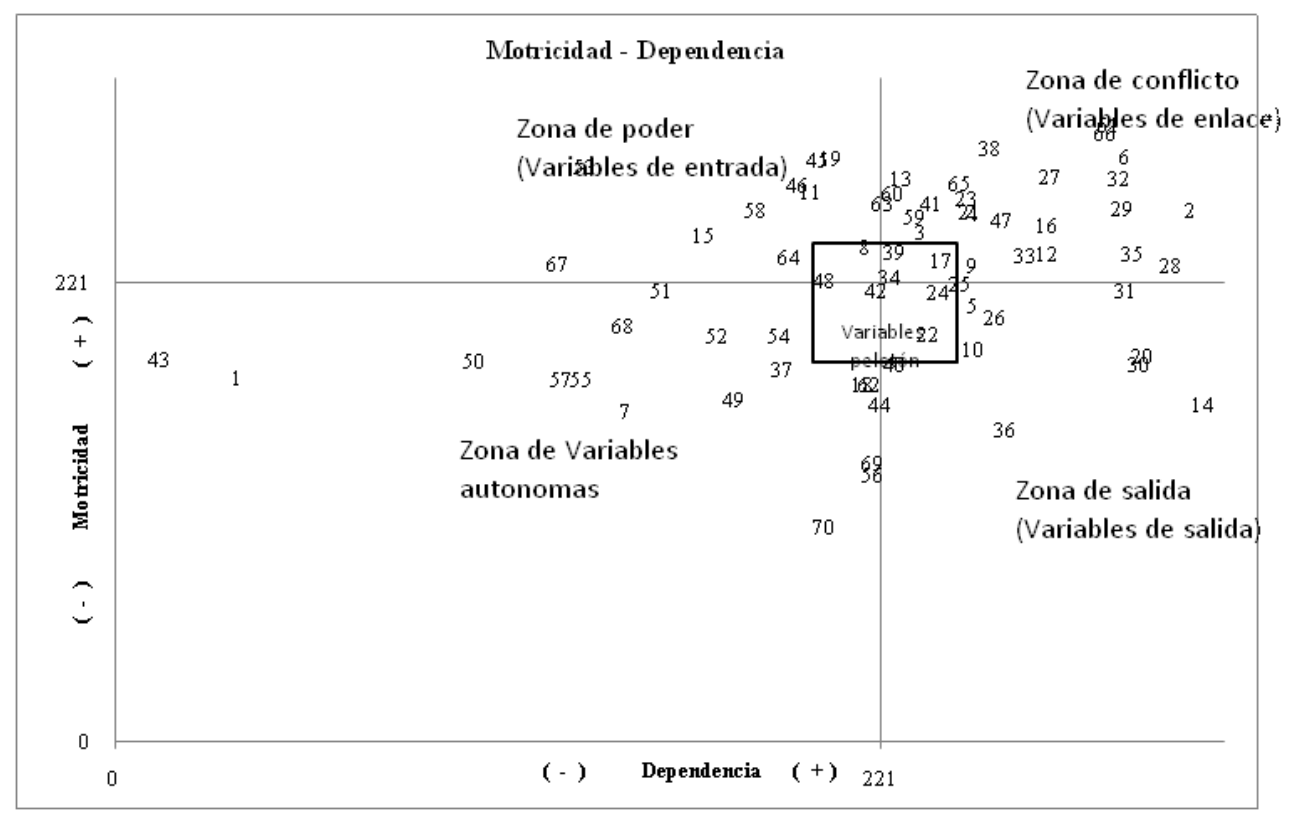

Figura 2: Eje cartesiano Motricidad-Dependencia

un inconveniente a la hora de tomar decisiones y de implementar cambios en las actividades productivas primarias de la región, dependiendo si son tierras privadas, fiscales o tomadas ilegalmente. Todas estas variables influyentes se tendrán en cuenta en la elaboración de escenarios.

\begin{tabular}{|l|l|}
\hline $\mathbf{N}^{\mathbf{p}}$ & \multicolumn{1}{|c|}{ Variable } \\
\hline 11 & Valor agregado \\
15 & Nivel tecnológico \\
19 & Explotación no sustentable \\
45 & Estabilidad económica \\
46 & Demanda de la producción primaria \\
53 & Ética \\
58 & Asociaciones de productores \\
64 & Escuelas de oficio \\
67 & Dominio de las tierras \\
\hline
\end{tabular}

Figura 3. Variables de la "zona de poder" (Segundo cuadrante)
Las variables de la Figura 4 tienen al mismo tiempo alta motricidad y alta dependencia, lo que imprime la importancia es su condición de enlace, que al ser influyentes sobre la zona de salida son a la vez influidos por las variables de poder. Sobre estas variables habrá que ejercer la presión de cambio deseado para lograr el escenario alterno positivo, por lo que también formarán parte de las variables clave.

En el cuarto cuadrante (Figura 5) se ubican las variables que tienen una alta dependencia y son muy poco motrices; por lo que son consecuencia de las variables explicadas en los dos cuadrantes anteriores. La "articulación regional", como las variables de "infraestructura de comunicación", la "producción de

2 I 0 Carla Narbaiza - Alejandro Schweitzer 


\begin{tabular}{|l|l|l|l|}
\hline $\mathbf{N}^{\mathbf{0}}$ & \multicolumn{1}{|c|}{ Variable } & $\mathbf{N}^{\mathbf{0}}$ & \multicolumn{1}{|c|}{ Variable } \\
\hline 2 & Planificación y Gestión del Territorial & 32 & Nivel de protección y conservación del recurso \\
4 & Política & 33 & Recursos hidricos \\
6 & Visión a largo plazo & 35 & Deterioro del paisaje \\
9 & Infraestructura para los sistemas productivos & 38 & Información ambiental \\
12 & Reciclado y utilización de desperdicios en la producción primaria & 41 & Exportaciones de materia prima sin manufactura \\
13 & Manejo de los recursos naturales & 47 & Oferta de la producción primaria \\
16 & Transferencia y adopción de tecnologías disponibles disponibles & 59 & Acuerdo entre actores \\
21 & Explotación de hidrocarburos & 60 & Hábitos del prouctor \\
23 & Explotación minera & 61 & Valoración del recurso \\
27 & Producción ganadera & 65 & Capital cultura \\
28 & Producción agrícola & 66 & Valoración del territorio \\
29 & Explotación forestal & & \\
\hline
\end{tabular}

Figura 4. Variables de la "zona de conflicto" o "variables de enlace" (Tercer cuadrante)

forraje", la "competencia entre usos del suelo", así como la actividad acuícola, son consecuencia de las variables en conflicto y las de poder. Por lo que un cambio en estas últimas provocará un cambio significativo en estas variables también, influyendo en los "riesgos ambientales" en el deterioro del "recurso natural" y en la "fauna silvestre nativa", a su vez que podrán generar una mayor conciencia de la importancia de la "certificación de denominación de origen patagónico", para potenciar la actividad productiva.

\begin{tabular}{|r|l|}
\hline $\mathbf{N}^{\mathbf{P}}$ & \multicolumn{1}{|c|}{ Variable } \\
\hline 5 & Articulación regional \\
10 & Infraestructura de comunicación (vial \\
& y portuaria) \\
14 & Producción de forrajes \\
20 & Competencia entre usos del suelo \\
26 & Acuicultura \\
30 & Riesgos ambientales \\
31 & Deterioro del recurso natural \\
36 & Fauna silvestre nativa \\
40 & Certificación de denominación de \\
& origen patagónico \\
\hline
\end{tabular}

Figura 5. Variables de la "zona de salida" (Cuarto cuadrante).

\begin{tabular}{|l|l|l|l|}
\hline $\mathbf{N}^{\mathbf{P}}$ & \multicolumn{1}{|c|}{ Variable } & $\mathbf{N}^{\mathbf{0}}$ & \multicolumn{1}{|c|}{ Variable } \\
\hline 1 & Posicionamiento geográfico & 32 & Nivel de protección y conservación del recurso \\
7 & Inmigracion & 33 & Recursos hidricos \\
18 & Estándares sanitarios & 35 & Deterioro del paisaje \\
37 & Especies introducidas invasoras & 38 & Información ambiental \\
43 & Economía internacional & 41 & Exportaciones de materia prima \\
49 & Marco legal de promoción económica & 47 & Oferta de la producción primaria \\
50 & Regalías & 59 & Acuerdo entre actores \\
51 & Impuesto y aranceles & & \\
52 & Fiscalización & 60 & Hábitos del prouctor \\
54 & Arraigo e identidad cultural & 61 & Valoración del recurso \\
55 & Población urbana mayoritaria & 65 & Capital cultura \\
56 & Nivel de conflictividad social & 66 & Valoración del territorio \\
57 & Asociaciones civiles & & \\
62 & Calficación de la mano de obra & & \\
68 & Ocupación ilegal de tierras & & \\
69 & Demanda de trabajo & & \\
70 & Oferta de trabajo & \\
\hline
\end{tabular}

Figura 6. Variables autónomas (Primer cuadrante) y variables pelotón 
En la zona de las variables-autónomas (Figura 6) se encuentran las variables que tienen baja motricidad y muy poca dependencia, por lo que estas no se encuentran solidarias con las demás variables en la conformación del sistema y no se tendrán en cuenta para elaborar los escenarios. Estas variables, si bien son importantes y fueron mencionadas por algunos actores, en el análisis integral del sistema de desarrollo territorial para la región no se articulan con las demás variables.

En el gráfico cartesiano (Figura 2), en las líneas promedio entre los cuadrantes se ubicaron las variables "capacidad técnica", "la actividad pesquera" y "la actividad turística". Estas tres variables no aparecen como relevantes para los escenarios, debido a su ubicación imprecisa en la gráfica, ya que se podría suponer que estas no tienen sobre las demás variables una influencia bien determinada. Lo mismo ocurre con las variables ubicadas alrededor del cruce de las líneas promedio, las cuales, al encontrarse poco definidas y al no tener casi relevancia para el sistema, son denominadas "variables pelotón" (Figura 6).

Las variables clave seleccionadas fueron las ubicadas en la zona de poder (Figura 3) y en la zona de conflicto (Figura 4), y en total suman 32 variables. Las variables «clave» fueron aquellas que al ser altamente motrices resultaron igualmente dependientes, porque estos factores son mucho más influyentes sobre los demás $\mathrm{y}$, al mismo tiempo, más fácilmente controlables por los actores sociales.
Actuar sobre estas variables podría indicar un mayor cambio al Sistema Regional, si se hace en la dirección correcta para lograr un escenario alterno positivo.

Una vez obtenidas las variables clave se aplicó el análisis morfológico de Zwicky, que consiste en la exploración de todas las dimensiones relevantes del tema bajo consideración; se sugieren atributos de un objeto o concepto (en este caso de las variables clave) y luego se procede a imaginar posibles variaciones para cada uno de los posibles escenarios alternos. El supuesto central del análisis morfológico es que alrededor de cualquier concepto existente se encontrarían ocultas una multiplicidad de variaciones, algunas potencialmente innovadoras.

\section{Resultados}

Según el método de Zwicky, el total de escenarios o de combinaciones posibles de las diferentes variables y sus opciones es el resultado combinatorio de todas las opciones por variable; este producto es el denominado "Espacio Morfológico", y no es presentado aquí dado el reducido espacio de la publicación. Privilegiando ciertas preferencias y eliminando algunas incompatibilidades, la elaboración de los escenarios se basó principalmente en aquellos resultados en los que predominó: a) la no variación de la situación actual (tendencial); b) situación alterna no deseada, cuando las variables que no atenúan o modifican los conflictos detectados se tornan dominantes, y c)

212 Carla Narbaiza - Alejandro Schweitzer 
situación alterna deseada, cuando se potencian las variables en pro del desarrollo territorial sostenible.

\section{Escenario tendencial}

Este escenario surge de la hipótesis de que la situación actual continúe dominando las variables clave, por lo que en el futuro se prevén cambios no muy alentadores. En tal caso el escenario esperable en un futuro podría ser:

- Situación de mayor incertidumbre en las actividades productivas primarias, principalmente debido a que la planificación y gestión territorial actual resulta teórica e irrelevante, lo que significa que, en el mejor de los casos, se plantea algún tipo de planificación en algunos sectores, pero está costando llevar a la práctica acciones precisas que determinen el cambio. Situación dominada por "políticas cortoplacistas" de solución momentánea, lo que sin una posterior planificación determinará nuevas y aceleradas situaciones de caos. Esta situación política, se encuentra en sintonía con las actividades productivas que no tienen una "visión de largo plazo".

- Disminución de la explotación de hidrocarburos y de minería. Según se pudo ver, son las actividades basadas en la explotación de recursos naturales que mayor dinamismo económico y social tienen, por lo tanto, su cortoplacismo de alto rendimiento económico actual, pero desarticulado del sistema regional, puede causar serios riesgos para el futuro de la región, económica, social y ambientalmente, ya que quedará un territorio desabastecido de los recursos naturales que derivaban en una actividad económica rentable y de alto impacto social. Esto aumentará la incertidumbre de una sociedad muy dependiente de esta actividad, que desaparece a causa del agotamiento del recurso.

- El mismo "manejo extractivo" de los recursos se da en las otras tres actividades que resultaron como variables en conflicto según la matriz de motricidad-dependencia: la ganadería, la agricultura y la forestal.

- Pérdida de capacidad de desarrollo endógeno. El bajo nivel tecnológico aplicado a la producción primaria, principalmente debido a una baja adopción de tecnologías hoy disponibles, a la información ambiental insuficiente y a la elemental infraestructura con la que cuentan los sistemas productivos, deviene en un escaso valor agregado, que termina limitando el tipo de oferta de la producción primaria, condicionando la utilización de los recursos naturales a la exportación de materia prima sin manufactura, o a un escaso mercado interno. De esta manera también se seguirán perdiendo mercados, que siendo más exigentes en calidad y cantidad, dejan de lado estos productos. Esta dependencia de mercados externos y la falta de un 
mercado interno condicionan la estabilidad económica, haciéndola momentánea e incierta.

- Además, la incertidumbre económica influye en la ausencia de "ética" por parte de los productores y del Gobierno, que genera una situación de aislamiento y desconfianza que impide el acuerdo entre estos actores, lo que repercute en que las asociaciones de productores existentes sean escasas e inactivas, de poca influencia en el desarrollo de las actividades productivas. Por lo tanto, no lograrán en un futuro concretar los leves impulsos de ordenamiento de las actividades productivas y de conservación de los recursos que se dan en la actualidad.

- Disminución de las tierras fiscales. Deterioro de las tierras de propiedad privada debido al mal manejo de los recursos, disminución del valor de la tierra.

- Deterioro del recurso natural y del paisaje. El productor, al tener incertidumbre en el presente, tiene una baja adopción de tecnologías puestas a favor de un futuro, más incierto aún, primando lo económico. Así, los hábitos del productor, adaptados a un sistema de desarrollo productivista, se tornan en una variable que tiene mucha incidencia en la "explotación no sostenible", lo que llevará a una disminución en la disponibilidad de recursos para la producción, no habrá en un fututo exportaciones y tampoco ingresos económicos derivados de la producción primaria de estas actividades, sumado a un paisaje empobrecido de recursos naturales.

- La inestabilidad económica y la falta de acuerdos concretos y duraderos entre los actores sociales inciden en el bajo nivel de protección y de conservación de los recursos. Se suma a esta situación el escaso reciclaje y utilización de los desperdicios en la producción primaria, que aumentan el deterioro del recurso natural.

- Sin intensificación de actividad ganadera y agrícola. Junto con el escaso nivel tecnológico se encuentra el mal aprovechamiento de los recursos hídricos disponibles, por lo que en un futuro no se espera una sistematización de ellos que aporte a la intensificación de las actividades agrícolas y ganaderas, generando un cambio en el sistema productivo extensivo.

- Capital cultural desaprovechado y estancado. Aunque en la actualidad la región cuenta con un capital cultural (se podría decir de un nivel medio), la situación no prevé una mejora sustancial en este sentido cultural. Aunque se vislumbra una oportunidad en la instalación de una nueva institución académica, la Universidad Nacional de Tierra del Fuego, para potenciar este capital cultural será necesario que se oriente la formación académica a enriquecer la mano de obra y de técnicos para el cambio en dirección de un mejor

2 I 4 Carla Narbaiza - Alejandro Schweitzer 
aprovechamiento de los recursos disponibles, basado en la valoración integral del territorio.

Esta situación determina que la explotación de los recursos está muy débilmente considerada dentro de las potencialidades de desarrollo para la región. Por más que hoy se pueden ver algunos intentos de evolución en la toma de conciencia al respecto de la situación en la que están los recursos naturales y las actividades primarias que de ellos dependen, para el futuro, de no cambiar en forma integral todas las variables descritas:

- la economía de la región Patagonia Sur tendrá que basarse en otro tipo de actividades, industriales, quizás, de finanzas, de comercio etc. Pero difícilmente se podrá basar en la producción primaria, dado que el mal manejo de los recursos de hoy y la nula planificación para un desarrollo integral futuro, están actuando en detrimento de las potencialidades basadas en la utilización de los recursos naturales.

- la pérdida de competitividad de la economía regional, sumada a la falta de organización y control del territorio producirá un círculo vicioso de declinación y falta de valorización integral de los recursos.

- Como consecuencia de lo planteado se podría prever igualmente un deterioro marcado de la calidad de vida y un aumento de la marginalidad, debido a la retracción de las oportunidades, que podría generar la promoción económica, y a la falta de concreción de nuevas oportunidades de desarrollo local.

\section{Escenario alterno de tendencia negativa}

La situación actual y su tendencia no son muy positivas, según se describe en el escenario anterior. Sin embargo, la situación puede ser peor si no se aprovechan las oportunidades que actualmente existen en la región. Resulta difícil determinar cuál podrá ser la tendencia de algunas de estas acciones, ya que depende del camino que se siga. Aunque en la actualidad se presentan oportunidades y corre la «sensación» de estar haciendo las cosas bien, pensando un futuro, si se toma el camino equivocado, debido principalmente a la falta de coordinación e integración dentro del territorio, podría traer aun consecuencias peores, $\mathrm{y}$, en tal caso, el escenario imaginario posible sería:

- Vuelta a empezar con los pocos recursos (naturales, económicos y culturales), con políticas que continúan siendo de corto plazo y con una planificación y gestión desestimada, considerada inútil para el desarrollo territorial debido a no haber cumplido con los objetivos y acciones planteadas en el pasado.

- Sin explotación primaria, debido a los escasos recursos naturales. Por lo 
tanto, no se podrá hablar de valor agregado de este tipo de producción, y menos de adopción de tecnologías en una actividad en total depresión, con una infraestructura en continuo detrimento.

- Sin estabilidad económica. Lo que acentúa la situación de caos y no permite una reactivación de la producción a través de la incorporación de tecnologías que permitan utilizar los escasos recursos disponibles.

- Alta demanda de productos primarios, debido a la escasa oferta mundial, oportunidad desaprovechada por la región para abastecer un mercado exigente y sin oferta.

- Sin exportación de materia prima, debido a que no hay oferta de ella.

- En una total falta de ética; no existen asociaciones de productores y los actores bregan por sus propios intereses.

- Abandono de establecimientos productivos y, como consecuencia, aumento de la ocupación ilegal de las tierras y de las tierras fiscales, debido a la falta de planificación y utilización de estas en actividades productivas planificadas.

- Los pocos productores existentes siguen manteniendo sus hábitos. A pesar de los resultados obtenidos, no han aprendido que la visión productivista los llevó a una situación límite en la actividad primaria.

- Los pocos recursos naturales disponibles tienen el valor de protección y se encuentran en áreas donde no se permite la explotación. Estas áreas deberían utilizarse para el fin que fueron creadas, como reserva genética y diversidad de especies, que permitan la reinstalación y recuperación de las áreas degradadas. Pero la falta de información ambiental hace imposible una utilización eficaz de estos recursos.

- Los recursos hídricos se encuentran contaminados y por lo tanto no se pueden utilizar para consumo ni para impulsar una producción intensiva.

- El paisaje se encuentra en un deterioro total, producto de la escasa valoración en el pasado.

- Debido a esta situación límite, existe una fuerte protección de los recursos en nivel normativo, y una revalorización del territorio y de los recursos perdidos. Sin embargo, el caos presente lleva a que sea más difícil aún poner en práctica las limitaciones normativas, bajo un capital cultural empobrecido, como consecuencia de un pasado de desestimación de este capital para el territorio.

\section{Escenario alterno positivo}

Este escenario (Figura 7) se podría dar si se potencian y planifican aquellos 
atributos de las variables clave, de la siguiente manera positiva e integral:

- La planificación y la gestión participativa parten de un acuerdo multisectorial entre los diferentes actores: el Estado, la sociedad, las organizaciones civiles y los productores. Se implementa una política de largo plazo que supera a los políticos de turno.

- Esta política basa el desarrollo regional sostenible en la valorización del territorio, mediante un alto nivel tecnológico y de infraestructura adecuada a una utilización racional de

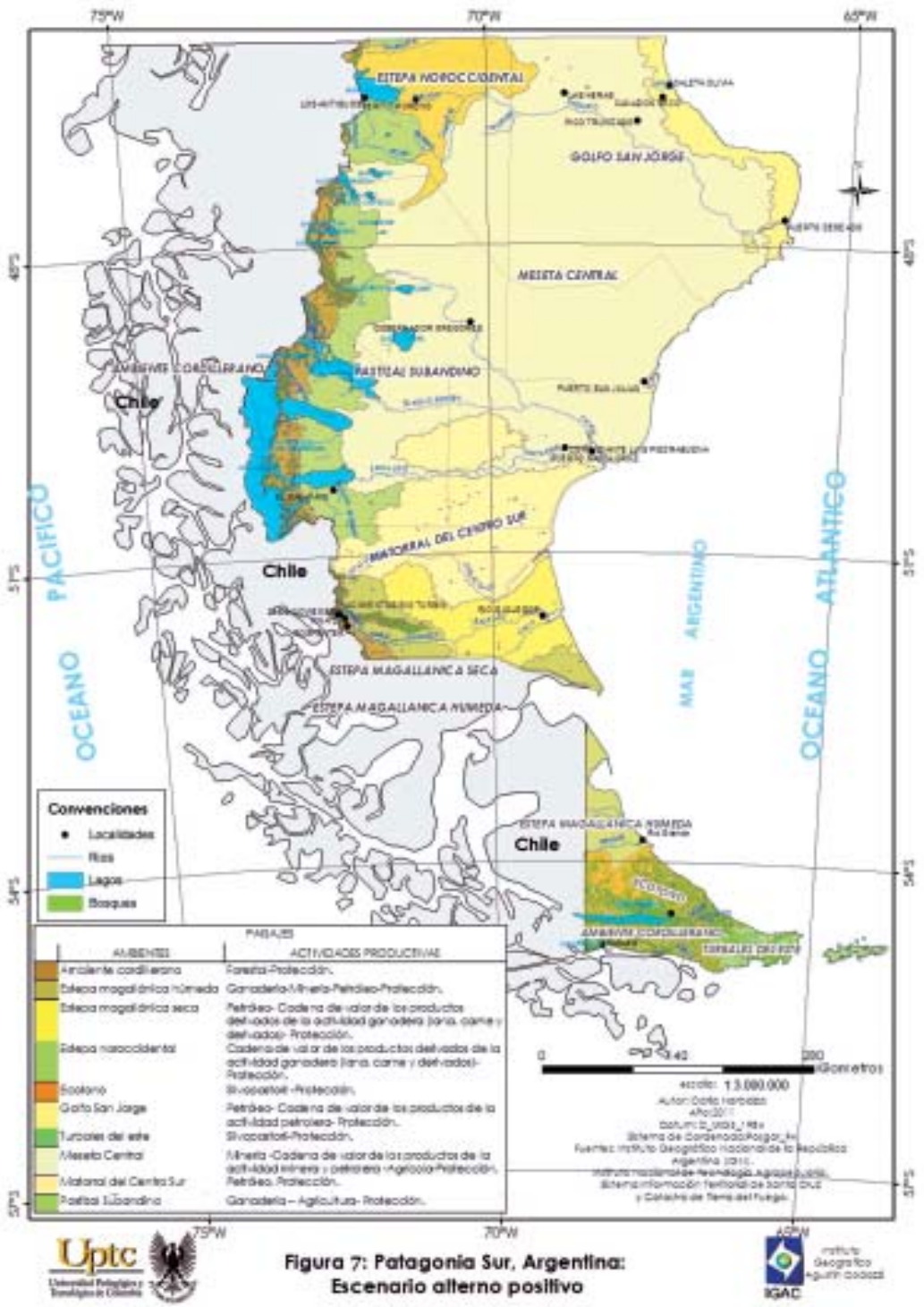

Perspect. geogr. Vol. 15. Año 2010, pp. 201-224 1217 
los recursos naturales, potenciando las actividades primarias y la cadena de valor en la integración regional.

- La actividad hidrocarburífera se desarrolla como actividad primaria de alto impacto, proyectando los beneficios económicos en un futuro planificado, aumentando paulatinamente el procesamiento de la materia prima en la región y generando nuevos mercados, más elevados en la cadena productiva, y autoabasteciendo el desarrollo regional, que necesitará un gran impulso energético para ponerse en marcha. El valor agregado a esta producción y la utilización eficiente de los residuos de la actividad permitirá alargar la vida útil de estos recursos en un cambio energético que permita su paulatina sustitución, potenciando investigación y desarrollo en energías alternativas.

- La minería se desarrolla en una escala local de alto valor agregado, se potencia su cadena de valor en la región y se abren nuevos mercados, aumentando los ingresos económicos por valor y no por explotación en bruto. Joyería de excelencia para la utilización del oro y plata. Cadena de valor en la construcción para utilización de los áridos extraídos. Con alto nivel tecnológico se minimizan los impactos negativos sobre el ambiente, y con un aumento en el valor agregado se controla la extracción primaria.

- Se impulsa la intensificación de la actividad ganadera en el ambiente del pastizal subandino y en la estepa magallánica húmeda, donde las condiciones ambientales son propicias para un manejo racional y de aplicación de las tecnologías de manejo desarrolladas. En la zona de ecotono y en los turbales del este se impulsa la actividad silvopastoril, con manejo del bosque y del pastizal simultáneamente, asegurando la sostenibilidad del sistema. Se ubican las actividades de los eslabones más altos de la cadena de valor de los productos (lana, carne y derivados) en los ambientes más vulnerables a la desertificación, quitándoles así la presión que ejerce el ganado sobre ellos. Manejando estas grandes extensiones para su recuperación, se da un valor de conservación, impulsando otras actividades de menor extensión y de mayor impacto económico, incentivando la denominación de origen y la certificación de calidad, y abriendo mercados a nivel nacional e internacional de productos de calidad.

- La actividad agrícola se dará principalmente en los valles irrigados, potenciando la cadena de valor de los productos agrícolas regionales, impulsando la denominación de origen, la certificación, y abriendo mercados, al igual que las demás actividades. La horticultura se encuentra articulada al sistema regional, satisfaciendo principalmente un mercado interno amplio y en crecimiento.

- La actividad forestal se da principalmente en los bosques

218 Carla Narbaiza - Alejandro Schweitzer 
subandinos, utilizando los bosques nativos y la alta tecnología, e integrada al sistema regional de industrialización de la materia prima, impulsando la cadena de valor. Esto garantiza mayor ingreso económico, debido al agregado de valor, y disminuye la presión sobre el recurso, permitiendo la recuperación de las zonas degradadas por mal manejo pasado.

- En todos los ambientes existe un área de protección que mantiene y mejora las características de los mismos e impulsa la educación ambiental.

- La integración de las actividades productivas en la industrialización diversificada de materia prima permitirá contar con una mayor estabilidad económica derivada de un desarrollo diverso y planificado, que amortigua posibles inflexiones económicas externas al sistema regional.

- El aprovechamiento racional del recurso hídrico es fundamental a lo largo de todo el proceso productivo y la cadena de valor, desde las actividades primarias, para su intensificación, hasta las industriales de la cadena de valor.

- En el desarrollo de estas actividades, el cuidado y la valoración del paisaje son el fundamento a seguir.

- Este escenario se da en absoluta presencia de ética en todos los actores, con apertura al cambio por parte de estos, innovando en sus hábitos para generar el cambio propicio y solidario indispensable, a través de asociaciones.

\section{Análisis de los resultados}

Los primeros dos escenarios presentan una situación futura muy poco alentadora para la región. De continuar esta desarticulación regional entre actividades primarias y una mirada no centrada en las potencialidades productivas, planificando hacia dentro de la región, uno de estos escenarios, o uno muy similar, podría irse dando en muy poco tiempo.

Para alcanzar el escenario alterno a la situación negativa descripta, y para efectivizar ese escenario positivo tan exigente y de progreso regional presentado, está implícita en él la planificación de las actividades dentro del desarrollo territorial sostenible. Es fundamental contar con objetivos claros para alcanzar el escenario deseado, que permitan articular solidariamente las diferentes acciones para un mismo fin.

La oportunidad no está en abastecer la demanda de materia prima exigida por un mercado mundial cada vez más escaso en recursos, sino en dominar el mercado de los derivados de materia prima elaborados en la región a través de denominación de origen y de un uso racional de los recursos, que permita mantener la producción en el tiempo para abastecer ese mercado. Evitar la tentación de explotación irracional cortoplacista ante una mayor demanda será el reto más importante y más eficaz 
a la hora de dominar los mercados internos y externos. La industrialización de la materia prima resulta el potencial más importante por desarrollar en la región, la cual deberá cumplir con las más exigentes tecnologías de conservación del ambiente y optimización de los recursos escasos.

Ante un escenario de desarrollo territorial sostenible se puede hablar de calidad de vida; de potenciar un capital cultural latente y en continua superación; de una sociedad independiente en cumplimento de sus deberes y en pleno goce de sus derechos, y de una sociedad que ha logrado readaptarse a su naturaleza.

\section{Conclusiones}

\section{Pertinencia de la metodología}

La metodología utilizada resultó adecuada para un estudio como el realizado aquí, donde existe gran cantidad de variables por analizar, las cuales, al resultar de un diagnóstico ambiental y social, retrospectivo y de consulta a los actores principales, parecen tener la misma relevancia dentro del sistema. El análisis estructural permitió categorizar las variables y darle la importancia justa a cada una; a su vez calificó la influencia que ejercen unas variables sobre otras, permitiendo de esta manera presentar alternativas de planificación futura, teniendo en cuenta las variables clave para el desarrollo territorial de la región. Por su parte, la flexibilidad del análisis morfológico hizo posible tener conocimiento de la complejidad del sistema analizado, para poder considerarlo en su totalidad.

\section{Conclusiones generales}

Realizar la planificación y gestión territorial de manera integral, participativa y consensuada no limitará la ocurrencia de "eventualidades", mas sí podrá garantizar un replanteo continuo y dinámico de las acciones por desarrollar en pro de objetivos comunes, los que también pueden ir consolidándose mientras se transforman en nuevos objetivos por cumplir.

Es evidente que ante un escenario de desarrollo regional tan autosuficiente como el presentado, las políticas serán su base primordial, sobre todo en las relaciones de comercio internacional. El comercio internacional de los países desarrollados ha dominado siempre la demanda de materia prima para su manufactura en el exterior, y este tipo de impulso de desarrollo regional hacia adentro tendrá su contraataque en este mundo globalizado de conquista constante y dominio de unos pocos. Estas son algunas de las contingencias que este escenario deberá considerar para poder ser una realidad. 


\section{Literatura citada}

Andrade, L. 2010. Otoño en la estepa. Ambiente, ganadería y vínculos en la Patagonia austral. Buenos Aires: La Colmena.

Andrade, L. 2005. Sociología de la desertificación. Los productores ovinos de la Patagonia austral. Buenos Aires: Miño y Dávila.

Borla, M. y Vereda, M. (2006). Explorando Tierra del Fuego: manual del viajero en el fin del mundo (2. ${ }^{\mathrm{a}}$ ed.). Ushuaia: Utopías.

Cipriotti, P.; Collantes, M.; Rauber, R.; Escartín, C.; Braun, K. y Livraghi, E. (2008). Evaluación del estado de invasión de Hieraciumpilosella en Pastizales. Región del Ecotono. Informe Final. Ushuaia: Consejo Federal de Inversiones.

Collado, L. (2007). Informe diagnóstico bosques. Ushuaia: Tierra del Fuego, Antártida e Islas del Atlántico Sur. Ministerio de Economía. Dirección de Bosques Sistemas de Información Geográfica.

Collado, L. y Farina S. (2006). El bosque de Tierra del Fuego. Caracterización, regiones ecológicas, actividad forestal, problemáticas y desafios actuales. Ushuaia: Tierra del Fuego. Subsecretaria de Recursos Naturales. Dirección de Bosques.

De Jong, G. (2009). Geografía, método regional y planificación. Buenos Aires: Catálogos.

Del Valle, H.; Elisalde, N.; Gagliardini, A.; Milovich, J. (2004). Desertificación en la Patagonia. Guía geográfica interactiva de Santa Cruz. Obtenido el 16 de noviembre de 2010 desde http://www.inta.gov.ar/santacruz/info/documentos/teledet/ guisantacruz_portada.htm

Equipo de Gestión Económica y Social. (2008). Informe de Situación. Santa Cruz. Obtenido el 12 de diciembre de 2010, desde www.eges.com.ar.

Gabiña, J. (1999). Prospectiva y planificación territorial. Hacia un proyecto de futuro. México D.F.: Alfaomega.

García, J. (2006). Geografía regional. En: D. Hiernaux y A. Lindón (Eds.): Tratado de Geografia Humana, pp. 25-70. Barcelona: Anthropos.

Hernández, J. (2009). Resistencia territorial, cambios identitarios y confirmación de nuevos sujetos sociales en el campo. La experiencia de la unión campesina Emiliano Zapata Vive (Puebla-México). En: F. Velázquez y M. Ferro (Eds.): Las configuraciones de los territorios rurales en el siglo XXI, pp. 361-389. Bogotá: Pontificia Universidad Javeriana.

Perspect. geogr. Vol. 15. Año 20I0, pp. 201-224 22 I 
Instituto Argentino del Petróleo y del Gas. (2010). Obtenido el 18 de diciembre de 2010, desde http://www.iapg.org.ar/estadisticasnew

Instituto Nacional de Estadística y Censos. (2010). Censo Nacional de Población, Hogares y Viviendas 2010. Datos provisorios. Obtenido el 18 de diciembre de 2010, desde http://www.censo2010.indec.gov.ar/

López, R. y Cervantes J. (2002). "Unidades del paisaje para el desarrollo sustentable y manejo de los recursos naturales”. Cultura Estadística y Geográfica, 20: 43-49. México: Instituto Nacional de Estadística y Geografía.

Martínez Llaneza, D. (2010). La evolución económica reciente de la provincia de Santa Cruz. Río Gallegos: Universidad Tecnológica Nacional.

Massiris, A. (2006). Políticas latinoamericanas de ordenamiento territorial: Realidad y desafios. Tunja: Universidad Pedagógica y Tecnológica de Colombia.

Max-Neef, M.; Elizalde, A.; Hopenhayn, M. (1996). Desarrollo a escala humana: una opción para el futuro. Medellín: Fundación DagHammarskjold.

Méndez, E. (2006). Geografía actual: espacio geográfico, territorio y campos de acción. Mérida: Instituto de Geografía y conservación de recursos naturales. Universidad de Los Andes.

Ministerio de Relaciones Exteriores de la República de Argentina. (2010). Comercio Internacional y Culto. Subsecretaría de Desarrollo de Inversiones. Prosperar. (2010). Argentina. Obtenido el 12 de diciembre de 2010 desde http:// prosperar.gov.ar.

Mojica, F. (2005). La construcción del futuro. Concepto y modelo de prospectiva estratégica, territorial y tecnológica. Bogotá: Universidad Externado de Colombia.

Naranjo, F. (2006). "El paisaje y su utilidad para la ordenación del territorio". Geocalli: Cuadernos de Geografía, 7(14): 69-126. México: Universidad de Guadalajara.

Paruelo, J.; GolluscioI, R.; Jobbágy, E.; Canevari, M. y Aguiar, M. (2006). "Situación ambiental en la estepa patagónica”. En: A. Braun; U. Martínez Ortiz; M. Acerbi y J. Corcuera (Eds.): La situación ambiental argentina, pp. 302-320. Buenos Aires: Fundación Vida Silvestre Argentina.

Programa de las Naciones Unidas para el Desarrollo. (2009). Manejo sustentable de ecosistemas áridos y semiáridos para el control de la desertificación en la Patagonia. Obtenido el 17 de noviembre de 2010, desde http:// gefpatagonia.ambiente.gov.ar/.

222 Carla Narbaiza - Alejandro Schweitzer 
Quargnolo, E. (Responsable). (2007). Determinación de la "brecha tecnológica" existente en los sistemas de producción ovina de la Patagonia austral. Identificación de los puntos críticos e impacto económico, social y ambiental de la aplicación de la tecnología disponible recomendada para el manejo extensivo en el extremo sur de Santa Cruz. -Informe final-. Río Gallegos: Instituto Nacional de Tecnología Agropecuaria. Santa Cruz.

Reátegui, R. (2008). Fundamentos del desarrollo sostenible. Material correspondiente al curso de posgrado "Bases fundamentales del desarrollo sostenible". Bolivia: Centro Boliviano de Estudios Multidisciplinarios.

Sánchez, J. (1991). Espacio, economía y sociedad. Madrid: Siglo XxI.

Santa Cruz. Agencia de Desarrollo de Inversiones. (2004). Información socioeconómica. Obtenido el 18 de diciembre de 2010, desdewww.inversiones.gov.ar

Sarobe, J. 1999. La Patagonia y sus problemas (3. ${ }^{a}$ ed.). Buenos Aires: Estudios Unión para la Nueva Mayoría.

Schiavini, A. (2007). "Aves y mamíferos de Tierra del Fuego". En: H. J. Paileleo (Coord.): Patagonia Total, pp. 797-834. (s.1.): Barcel Baires.

Schorr, A. y Segui, M. F. (2008). Zonas agroeconómicas homogéneas. Patagonia Sur: Chubut, Santa Cruz y Tierra del Fuego. Santa Cruz: Instituto Nacional de Tecnología Agropecuaria Santa Cruz.

Schweitzer, A. (2004). Espacios de planificación y territorios a la deriva en la provincia de Santa Cruz. Trabajo presentado en V Jornadas Patagónicas de geografía área temática economías regionales. Río Gallegos, Argentina.

Schweitzer, A. (2008). Otra Patagonia austral es posible. Proyectos de territorio entre la emergencia y la urgencia. Proyección 5: Ordenamiento Territorial en Argentina. Obtenido el 14 de marzo de 2010, desde www.proyección.cifot.com.ar.

Schweitzer, A. (2009). Plan de Ordenamiento y Desarrollo Territorial -PODT-. Diagnóstico Territorial. Informe final. Río Gallegos: Consejo Federal de Inversiones.

Sepúlveda, S.; Rodríguez, A.; Echeverri, R. y Portilla, M. (2003). El enfoque territorial del desarrollo rural. San José de Costa Rica: Instituto Interamericano de Cooperación para la Agricultura.

Tricart, J. y Kilian, J. (1982). La eco-geografía. La ordenación del medio natural. Barcelona: Anagrama.

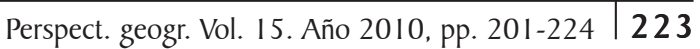


Troitiño, M. (2006). "Ordenación del territorio y desarrollo territorial: La construcción de las geografías del futuro". Geocalli: Cuadernos de Geografía, 7(14): 17-68. México: Universidad de Guadalajara.

Williams, M. (2006). Un diseño de políticas públicas para el desarrollo sostenible de las pequeñas y medianas empresas ganaderas de la provincia de Santa Cruz. Río Gallegos: Instituto Nacional de Tecnología Agropecuaria Santa Cruz.

Williams, M. (2009). La situación del sector ovino en Santa Cruz: análisis y reflexiones. Río Gallegos: Instituto Nacional de Tecnología Agropecuaria Santa Cruz.

Recepción: 20 de septiembre de 2010

Aprobación: 02 de noviembre de 2010

224 Carla Narbaiza - Alejandro Schweitzer 